\title{
The second Team Haemophilia Education Meeting, 2016, Frankfurt, Germany
}

Erik Berntorp ${ }^{1}$, Yesim Dargaud ${ }^{2}$, Daniel Hart ${ }^{3}$, Sébastien Lobet ${ }^{4}$, Maria Elisa Mancuso ${ }^{5}$, Roseline d'Oiron ${ }^{6}$, David Perry ${ }^{7}$, Debra Pollard ${ }^{8}$, Marijke van den Berg ${ }^{9}$, Jan Blatný ${ }^{10}$, Hervé Chambost $^{11}$, Andrea S. Doria ${ }^{12,13}$, Pål André Holme ${ }^{14}$, Radoslaw Kaczmarek ${ }^{15}$, Lorenzo Mantovani ${ }^{16}$, Paul McLaughlin ${ }^{17}$, Lochana Nanayakkara ${ }^{18}$, Pia Petrini ${ }^{19}$, Thomas Sannié ${ }^{20}$, Edward Laane ${ }^{21}$, Raquel Maia ${ }^{22}$, Athina Dettoraki ${ }^{23}$, Anna Farrell ${ }^{24}$, Susan Halimeh ${ }^{25}$, Sayma Raza ${ }^{26}$, Stephanie Taylor ${ }^{27}$

${ }^{1}$ Skåne University Hospital, Lund University, Malmö, Sweden; ${ }^{2}$ Clinical Haemostasis Unit, Lyon Hospital, University of Lyon, Lyon, France; ${ }^{3}$ Barts and the London School of Medicine and Dentistry, Queen Mary University of London, London, UK; ${ }^{4}$ Service d'hématologie, Cliniques Universitaires Saint-Luc, Brussels, Belgium; ${ }^{5}$ Fondazione IRCCS Ca' Granda, Ospedale Maggiore Policlinico, Angelo Bianchi Bonomi Haemophilia and Thrombosis Centre, Milan, Italy; ${ }^{6}$ Centre for Haemophilia and Rare Congenital Bleeding Disorders, University Hospitals Paris-Sud, AP-HP Bicêtre Hospital, Le Kremlin-Bicêtre, France; ${ }^{7}$ Addenbrooke's Hospital, University of Cambridge, Cambridge, UK; ${ }^{8}$ Katharine Dormandy Haemophilia \& Thrombosis Centre, Royal Free Hospital, London, UK; ${ }^{9}$ Department of Health and Epidemiology, University of Utrecht, Utrecht, The Netherlands; ${ }^{10}$ Department of Paediatric Haematology, Children's University Hospital and Masaryk University, Brno, Czech Republic; ${ }^{11}$ Department of Paediatrics, La Timone Children Hospital, APHM and Aix-Marseille University, Marseille, France; ${ }^{12}$ Department of Diagnostic Imaging, The Hospital for Sick Children, Toronto, ON, Canada; ${ }^{13}$ Department of Medical Imaging, University of Toronto, Toronto, ON, Canada; ${ }^{14}$ Department of Haematology and Institute of Clinical Medicine, Oslo University and Oslo University Hospital, Rikshospitalet, Norway; ${ }^{15} \mathrm{Hirszfeld}$ Institute of Immunology and Experimental Therapy, Wroclaw, Poland; ${ }^{16}$ Public Health, CESP-Center of Public Health Research, University of Milano-Bicocca, Milan, Italy; ${ }^{17}$ Department of Physiotherapy, Katharine Dormandy Haemophilia Centre, Royal Free Hospital, London, UK; ${ }^{18}$ Department of Restorative Dentistry, Barts Health NHS Trust, London, UK; ${ }^{19}$ Department of Paediatrics, Karolinska University Hospital, Stockholm, Sweden; ${ }^{20}$ Association Française des Hémophilies (AFH), Paris, France; ${ }^{21}$ North Estonia Medical Centre, Tallinn, Estonia; ${ }^{22}$ Paediatric Haematology Unit, Dona Estefânia Hospital, Lisbon, Portugal; ${ }^{23}$ Haemophilia Centre and Haemostasis Unit, 'Aghia Sophia' Children's Hospital, Athens, Greece; ${ }^{24}$ Bristol Royal Hospital for Children, Bristol, UK; ${ }^{25}$ Gerinnungszentrum Rhein-Ruhr (GZRR), Duisburg, Germany; ${ }^{26}$ Oxford University Hospitals NHS Foundation Trust, Oxford, UK; ${ }^{27}$ Oxford Haemophilia and Thrombosis Centre, Oxford University Hospitals Foundation Trust, Oxford, UK

\begin{abstract}
The first Team Haemophilia Education (THE) Meeting was held on 7-8 May 2015 in Amsterdam, The Netherlands. It aimed to promote the optimal care of patients with haemophilia through education of the multidisciplinary treatment team. This was achieved by reviewing the latest developments in haemophilia management, considering how these can be implemented in the clinic to improve patient care and providing a platform for networking and debate for all haemophilia treatment team members. The second THE Meeting was held on 19-20 May in Frankfurt, Germany, and participants included doctors, nurses, physiotherapists, patient representatives and data management staff from 20 different countries. Topics covered the role of the multidisciplinary team in delivering the best haemophilia care, challenges in the management of haemophilia across Europe, available clotting factor treatments, future treatments and the use of genetics in advising carriers of haemophilia. This report is a summary of the key developments in haemophilia care presented by various investigators and healthcare professionals at THE Meeting 2016.
\end{abstract}

Key words haemophilia; genetics; real-life data; laboratory assays; clotting factor concentrates; multidisciplinary

Correspondence Erik Berntorp, Skåne University Hospital, Lund University, Malmö, Sweden. Tel: +46705752496; Fax: +4640336255; e-mail: erik.berntorp@med.lu.se 


\section{Introduction}

\section{Erik Berntorp (Malmö, Sweden)}

The first Team Haemophilia Education (THE) Meeting was held on 7-8 May 2015, in Amsterdam, The Netherlands. The aim of the meeting was to promote optimal and seamless care of patients with haemophilia through education of the multidisciplinary treatment teams. The aim of this second meeting here in Frankfurt is to deduce the best care and to promote the integration of the multidisciplinary team in caring for patients with haemophilia through education. The Steering Committee has been enriched by a few new members this year from different disciplines and has worked closely together during this last year to plan this meeting. Topics covered in the 2016 meeting include unmet clinical needs, transition models of care, management of patient data and haemophilia carriers, and genetic aspects of haemophilia. In addition, there were several workshops that included the following: 'The differing roles of the multidisciplinary team in delivering the best possible care', 'What imaging at what time?', 'Physical activity as a treatment', 'Global assays in the clinical setting' and 'Dental procedures: demystifying risk and minimising haemostatic cover requirements'.

This report is a summary of the scientific developments in haemophilia care presented by various multidisciplinary care team members at THE Meeting 2016.

\section{Ice breaker session - centre presentations}

Edward Laane (Tallinn, Estonia), Raquel Maia (Lisbon, Portugal), Athina Dettoraki (Athens, Greece), Anna Farrell (Bristol, UK)

The European Association for Haemophilia and Allied Disorders (EAHAD) has endorsed a set of guidelines for optimal haemophilia care, but the type of treatment and availability of specialist health services vary among different countries. In this session, representatives from 11 selected centres across Europe (Estonia, Germany, Greece, Italy, Portugal and UK) presented their experiences in certain areas of haemophilia management, with the aim of facilitating discussion on how to improve patient care.

\section{Estonia}

In Estonia, the main challenges are for hospital management to understand haemophilia, resources for oncology vs. haematology departments, the procurement system that is a huge problem (who will have the profit?), gaps in education, the fact that by law the doctor has to see 21 patients per day which means only $20 \mathrm{~min}$ each consultation, the patients' motivation if they are not followed properly, who is the haemophilia specialist - is it every haematologist? In Potemkin village, PR for publicity is a huge problem, supporting parallel management above the haemophilia nurse for whom patients with haemophilia represent only $2.7 \%$ of the total working load of haematology patients. Of these $2.7 \%$, not all patients are seen, as some go directly to the ward to collect their coagulation factor. In addition, there are changes in leadership, which is the biggest challenge in our hospital. To overcome our problems, we joined in a partnership with the centre in Helsinki, Finland, to build the haemophilia team, as a result of which the haemophilia nurses and specialists have been educated better, and there is close cooperation with the patients' organisation. There are meetings about the laboratory and clinic and laboratory methods have been validated. The laboratory is now part of the World Federation of Hemophilia (WFH) quality control programme, and our centre's visibility in terms of EHC and EAHAD has improved. Furthermore, we are now able to prescribe coagulation factors.

\section{Portugal}

In our centre, haemophilia care is divided into adult and paediatric branches. Most patients receive home treatment. The benefits of our centre include the following: (i) all specialties (rheumatology, orthopaedics, dentistry, infectious diseases, general surgery, genetics, physical medicine, rehabilitation, etc.) are available; (ii) no major limitations with regard to access to recombinant or bypassing agents; (iii) independent facilities for paediatric/adult care, but very good team communication; (iv) reasonably good collaboration with local hospitals and primary care teams; (v) EUHANET certification; (vi) paediatric, multidisciplinary Joint Disease Surveillance Program since 2014.

On the other hand, our main challenges include at a national level: (i) lack of formal organisation/national coordinator and national registry (pending government approval); (ii) product acquisition is decided by each centre's administration (pressure to change products on a regular basis due to costs; different products available in different centres) although now there is a tendency for product acquisition at a national level. Locally, we need a more specialised team, dedicated facilities/hours for haemophilia care and dedicated healthcare professionals outside of the haematology/blood bank team.

\section{Greece}

The Haemophilia Centre and Haemostasis Unit in 'Aghia Sophia' Children's Hospital in Athens consists of four sections: clinic, education, laboratory and research. There are three doctors and three nurses, plus laboratory staff. The centre belongs to several registries: Comprehensive Care Centre (CCC)-EAHAD, PedNet, EuHADD, Insight and EN-RBD. In the unit, there are 600 patients with haemophilia and allied 
disorders, of whom 150 have haemophilia. The follow-up starts from the time of diagnosis until the age of $18 \mathrm{yr}$. About $60 \%$ of the total number of patients with haemophilia $(80 \%$ of severe cases) are on prophylaxis. Currently, six children have inhibitors. Most patients $(60 \%)$ are on home therapy. About half of the patients are from Athens and surrounding areas, and about $20 \%$ from the islands, which causes many problems for patients when ships, etc. are cancelled due to bad weather or even strikes from time to time. The remaining $28 \%$ come from areas $>200 \mathrm{~km}$ from Athens that also causes problems. There are concerns about availability of concentrates as a result of a limited budget. To overcome these problems, newly diagnosed children are treated at local hospitals - there is cooperation with paediatricians/nurses. Nevertheless, families are encouraged to deliver home therapy (education for venipunctures), and intensive training for venipuncture or Port-a-Cath access is given at the haemophilia centre, if required. Patients/parents are educated about early detection of bleeding signs, and they are given a stock of factor concentrate to have at home. In case of emergency, extra factor concentrates are delivered, even on vacation days. Depending on the clinical presentation, the patient is hospitalised locally or transferred to the centre in Athens if the situation is serious. All haemophilic children are examined at least once per year. There is $24 \mathrm{~h} / 365 \mathrm{~d} / \mathrm{yr}$ availability and guidance by phone, and there is close collaboration with families.

\section{UK}

Bristol CCC consists of adult and paediatric services. Bristol Children's Hospital is commissioned to provide a CCC service to children in the south-west region. Approximately 140 children are registered with Bristol Children's Hospital from across the region. Care is provided by a multidisciplinary team comprising of consultants, nurse specialists, physiotherapists, a psychologist and laboratory staff. Bristol is located at the top of the south-west UK region, which presents the challenge that the furthest away a patient can be from the centre is $290 \mathrm{~km}$ which can mean $5 \mathrm{~h}$ of travelling. The solution to this problem was to have 6-monthly outreach clinics established in five regional centres with consultant haematologist, nurse specialist and physiotherapist from $\mathrm{CCC}$, and the local team. Families across the region required training administering factor via venous access, to facilitate home treatment, so they worked with local community paediatric nursing teams to provide education in the home setting. Ongoing training and education is provided to nursing teams to enable this to occur. To provide acute physiotherapy assessment and treatment in the regional centres, named physiotherapists were identified in each regional centre, and ongoing education and training is provided for them in formal meetings, which are linked with the centre in Bristol. Another challenge was assessing/reviewing bleeds of patients within the region over the phone with no moving visual images, but this was overcome with the introduction of video telephony to review patients in the home setting.

\section{Summary of the session}

This session has highlighted the problems some haemophilia treaters face in different countries and the variation in standard of care between countries. Many countries have geographical problems with patients living a long way from the treatment centre. In the centre in Bristol, UK a team comprising a specialist, a nurse and a physiotherapist makes 10 visits a year to different parts of the region to see patients and their families. In Greece, there are problems with families living on the islands who need to visit the treatment centre, for whom there is not usually financial help to pay the travel costs for their annual appointments. Another problem in Greece could be delivery of factor concentrates, but this is sent out using couriers. In Estonia, there are problems with very limited appointment times for a doctor, a maximum of 20 min per patient. In Norway, there are much greater distances from the centre about $2700 \mathrm{~km}$ maximum - but even so all patients can reach the centre in about 2-3 h. In Sweden, it is a very similar situation. The issue of distance from the treatment centre is more of a problem when the patient/parent is first learning how to give infusions.

\section{Plenary session 1: There is no perfect centre: challenges in the management of haemophilia across Europe}

\section{Paul McLaughlin (London, UK)}

The aim of this session was to discuss the goals of haemophilia treatment and how different haemophilia treatment centres across Europe aim to reach these targets.

In 1965, France and Wolf (1) stated that 'Not providing physiotherapy for painful, bleeding, arthritic joints "would make schooling practically impossible and jeopardise the boys' future career"'. This is still the case today. In 2008, EAHAD published their principles of care for haemophilia centres. They stated that CCCs and HTCs should prevent bleeding, ensure long-term management of joint and muscle damage, manage complications from treatment, have experienced staff and provide a service appropriate to the local population within the context of physical and political geography, and population density and distribution. CCCs should have a dedicated physiotherapy service available, provide access to rheumatology and/or orthopaedic services, provide access to psychological services and follow up patients regularly. Haemophilia treatment centres (HTCs) should have access to the service of a local physiotherapist with an awareness of haemophilia problems and have a strong relationship with the rheumatology/orthopaedic services at the CCC. 
When looking at the overall goals of haemophilia care, they are met when access to treatment and care allows patients to achieve their potential in life and protects patients from complications of haemophilia and its treatment. They are achieved by access to safe and effective CFCs with comprehensive care delivered by a multidisciplinary team. There is a wide variability in gross domestic product per capita vs. FVIII per capita, with Sweden having the highest consumption, and Austria having the lowest in terms of FVIII (2). Before this meeting, participants were asked what clinical services were available in their centres. Eleven centres responded, and the least available services were pain management and rheumatology, while the most available were paediatric care and dentistry. This raises an interesting question about who should be responsible for pain management in persons with haemophilia $(\mathrm{PWH})-$ is this a specialist pain service issue, or one that Haemophilia centres should improve upon? Clinical concerns are how should pain be assessed and by whom? What is the patient's joint function, what are the treatment issues, what intervention should be given? Shared decision making is the key to finding these answers.

Another major issue is the ageing population of patients with their associated comorbidities. For example, in the UK, there are over 8300 persons with haemophilia A or B and, in the past $40 \mathrm{yr}$, there has been a threefold increase in over-60s with haemophilia A, and an eightfold increase in over-60s with haemophilia B. They have different needs to younger patients and require different assessments (Table 1).

In addition, all patients are susceptible to developing inhibitors, and those of middle age and older are also more likely to have acquired infection with HIV/hepatitis C.

The behaviours and choices of some patients can be heavily influenced by their family's personal experience of haemophilia. It is very important that the haemophilia care

Table 1 Needs of patients with haemophilia according to age group

\begin{tabular}{ll}
\hline Age group & Needs/expectations \\
\hline Younger patients & $\begin{array}{l}\text { Prophylaxis, and the monitoring/surveillance } \\
\text { of its success (medical/physically) } \\
\text { Fitting in } \\
\text { Sport, exercise, activity and injury } \\
\text { 'Normalcy' - expectation and 'medically induced' } \\
\text { Usually only 1-2 problem joints } \\
\text { University/work } \\
\text { Sport, exercise, health and injury } \\
\text { Joint damage } \\
\text { Functional ability/pain } \\
\text { Prospect of orthopaedic surgery } \\
\text { Widespread pain/musculoskeletal dysfunction } \\
\text { Social isolation/fear/expectations of such } \\
\text { Falls, injury, immobility } \\
\text { Surgery (orthopaedic and those } \\
\text { associated with old age) } \\
\text { Diseases of old age }\end{array}$ \\
\end{tabular}

team helps to overcome these deep-seated views. In addition, figures suggest that up to $50 \%$ of patients have chronic pain that is not well controlled. Patients struggle to tell the difference between pain caused by a bleed or arthropathy, and those with arthropathy have anxiety about the future. It is important that all those working with PWH develop therapeutic relationships and ask the right questions of the patients in their care. What matters most to them about their life with haemophilia, and the effects of haemophilia on their physical and mental well-being? A knowledge of this will enable care to be individually tailored and effective.

\section{Radoslaw Kaczmarek (Wroclaw, Poland)}

Poland has a population of over 38 million people, of whom more than 4300 have a bleeding disorder ( $>2600$ with haemophilia A or B). Poland is a success story in how it developed the availability of clotting factor concentrates. Prior to 2005, IU/capita/year was very low - around 1. But between 2005 and 2011, there was a vast improvement in availability, and in 2014, the IU/capita/year was about 5. This came about because in 2005, the Ministry of Health accepted the National Hemophilia Treatment Program 20052011. In 2008, it was agreed that all patients aged under $18 \mathrm{yr}$ of age should receive prophylaxis. In 2012, the Ministry of Health accepted the National Hemophilia Treatment Program 2012-2018. The 2012-2018 Program includes the development of a network of comprehensive care centres and improved access to prophylaxis in adults. Currently, there are two CCCs, both in the capital. One is certified; however, there are no certified haemophilia treatment centres. With improving access to CFCs, the need for aspects of care other than haemostasis grows more important in terms of, for example, orthopaedic surgery and physiotherapy. However, budgetary constraints, underpriced medical services and overcommitment to research vs. management are all issues that need to be addressed.

There was no HIV outbreak in Poland in the 1980s due to a cruel historical irony that clotting factor concentrates were not available in Poland at that time, but $95 \%$ of PWH born before 1991 are anti-HCV-positive and $77.3 \%$ are chronically infected (HCV-RNA-positive) (3).

To conclude, meeting the needs of patients with haemophilia requires comprehensive care. Haemophilia must be appropriately recognised and prioritised within the healthcare system. Partnership and good communication with the patient is crucial to improve performance and optimise care.

\section{Debate 1 - How special are patients with haemophilia - should they always fly business class?}

\section{Dan Hart (London, UK)}

As healthcare professionals, should patients with haemophilia be offered the same service as all other patients, or 
alternatively offered a more 'business class'-like service for their care? Such a service should be responsive, easily accessible with a more personalised oversight to ensure care episodes run smoothly. For someone about to undergo a hip replacement, for example, the haemophilia multidisciplinary team coordinates care, outlining a clear management plan that is circulated widely to surgeons, anaesthetists, haemophilia staff, laboratory staff and ideally documented in electronic medical records if available. The haemophilia team coordinates care under clear lines of responsibility, with both pre- and immediate post-operative plans, subsequent out-of-hours care and ensuring specialist review at weekends. The haemophilia team most often 'dictates' discharge planning, with a clear followup/management plan following discharge communicated clearly to the patient. The capacity to deliver such a service of haemophilia care depends on the individual centre and healthcare service. Patient satisfaction surveys and structured audit of the service can highlight areas for improvement towards such a 'business class' service.

An article by Colvin et al. (4) outlined the European principles of haemophilia care. It recommended that within each country, there should be a national organisation that oversees the provision of specialist CCCs that provide the entire spectrum of clinical and laboratory services. It also recommended a national register of patients along with collection of treatment statistics.

\section{Lorenzo Mantovani (Milan, Italy)}

In Italy, the second most expensive medicine expenditure in the country is factor VIII (FVIII). However, if the patient has an inhibitor, treatment costs considerably more (5), this expenditure is essential to provide the patient with a good quality of life. Quality of life is associated with the orthopaedic status of PWH, with both the EQ-5D and SF-36 scores declining as the orthopaedic joint status deteriorates (6).

In 2012, Schramm et al. carried out a study to determine the clinical condition of patients with haemophilia within Europe, as recommended by the European Commission. Patients were grouped according to per capita amount of clotting factor used in their region of residence in 2005: region 1: >5 IU; region 2: $2-5 \mathrm{IU}$ and region $3:<2 \mathrm{IU}$. It was found that paediatric and adult patients in region 3 had median numbers of three and eight annual joint bleeds, respectively, with worse joint scores compared to region 1 with zero and one bleed. Prophylactic therapy was used in only $31.3 \%$ children and $8.9 \%$ adults with severe haemophilia in region 3 (Czech Republic, Lithuania, Poland, Romania and Turkey) compared to $93.7 \%$ and $54.1 \%$, respectively, in region 1 capita (Austria, Denmark, France, Germany, Sweden and the UK) (7).

\section{Thomas Sannié (Paris, France)}

In France, more than $50 \%$ of the population is affected by a chronic disease. More than half of the population do not follow their prescription, resulting in a public health issue.
Doctors, nurses and physiotherapists are trained during their medical school to focus on biomedical issues. A pathology affects not only the biomedical, but also the patient's personal perception and the environment. For young haematologists, we should introduce into the curriculum the concept as the patient as a teacher. Healthcare providers need to base their knowledge not only on evidence-based medicine and protocols, but also on patients' preferences, quality of life and shared decision-making. Patients are special, and a mutually beneficial relationship needs to be agreed upon. There are two main participants in this: the expert of the disease, that is the healthcare provider, and the expert of living with a disease, that is the person living with a chronic disease, or a family caregiver. Patients' organisation and healthcare providers need to agree on conducting together regular audits of the haemophilia treatment centre.

Five case scenarios involving patients with haemophilia about to undergo various procedures were then presented, and the audience was invited to participate in voting how they should be managed. The consensus was that patients with haemophilia should always have the opportunity to fly 'business class', underlining the importance of centres aspiring to provide a specialised, personalised service to care for this group.

\section{Workshop sessions}

\section{What imaging at what time?}

\section{Andrea Doria (Canada)}

PWH are prone to episodes of joint haemorrhages. If left untreated, such bleeding can lead to synovitis and ultimately joint degeneration. Therefore, assessing the extent of joint damage is important, and imaging provides critical information that can be used to inform treatment choices to provide the best level of patient care. Among available imaging techniques, ultrasound and magnetic resonance imaging (MRI) are used for evaluation of early changes of joints of PWH, and X-rays for quantification of late changes (8). MRI is considered the reference standard imaging method. It enables visualisation of the entire joint through individual slices providing detailed assessment of soft tissues (blood degradation products, synovial hypertrophy and fluids), cartilage and bone marrow. Ultrasound, on the other hand, provides a compartmentalised view of the joint through individual slices and presents with limitations for visualisation of the central aspect of the joints due to the limited penetration of the ultrasound beam with high-frequency transducers. As a result, it is accurate for assessing soft tissues along the periphery of the joint, but has variable accuracy for diagnosing osteochondral tissues (9). In contrast to the other two 
techniques, radiography bears radiation and provides information on structural bone damage through overlapped planes. With this technique, soft tissues are poorly assessed, and cartilage can only be indirectly evaluated by joint space narrowing. Considering the different diagnostic capabilities of different imaging techniques, the best timing for starting imaging of joints of PWH depends on the available prophylaxis scheme, the clinical question that should be answered and the patient's age at the time of first bleed(s) due to the risk of anaesthesia when performing MRI in young patients.

\section{Physical activity as a treatment}

\section{Sébastien Lobet (Belgium)}

Physical activity is important in both prevention and treatment of some of the major conditions of our time, including cardiovascular disease, diabetes, cancer, hypertension, obesity, osteoporosis and depression (10). It is recognised that properly managed exercise and participation in appropriate sports can be highly beneficial for PWH (11). However, exercise in PWH is associated with specific challenges, with injuries and potential bleeds being possible drawbacks. However, correctly managed participation can improve activity participation and physical performance, increased social inclusion and consequently higher self-esteem, as well as social adaptation. In this context, exercise therapy should be promoted in PWH. The term 'exercise therapy' (or exercise medicine) describes an activity prescribed by a physician, planned and dosed by physiotherapists or coaches and performed by the patient in a group or alone. This is important and should be as an entire part of the treatment for older $\mathrm{PWH}$, as with population ageing, exercise therapy may be an important means of reducing disability and increasing the number of older people living independently.

\section{Dental procedures: demystifying risk and minimising haemostatic cover requirements}

\section{Lochana Nanayakkar (UK)}

It is important to raise awareness of the importance of dental care and acknowledge that oral bleeding is not a symptom of a bleeding disorder but could be a symptom of a treatable dental condition (12).

\section{Plenary Session 2 - Update on clotting factor concentrates}

\section{Maria Elisa Mancuso (Milan, Italy)}

This presentation was divided into current treatment paradigm and new treatment approaches.

\section{Current treatment paradigm}

The primary aim of care is to prevent and treat bleeding episodes by administering replacement therapy. Acute bleeds should be treated as soon as possible (within $2 \mathrm{~h}$ ). Continuous prophylaxis is the gold standard treatment, with the primary aim of prevention of arthropathy. Additional aims are an active lifestyle and good quality of life. Where appropriate and possible, replacement therapy should be delivered in the form of home treatment. Patients are best managed in a comprehensive care setting as suggested by the WFH and the EAHAD (4, 13).

Treatment is currently given using either recombinant or plasma-derived factor concentrates. Both are still administered by the intravenous route and are costly. Concentrates can be given either prophylactically or on-demand, and both are safe to use. Treatment needs to be tailored to the individual patient, taking into account their lifestyle and needs. There is still a subgroup of patients, that is those with inhibitors, for whom prophylaxis as intended for non-inhibitor patients is not possible.

Issues with current treatment are that prophylaxis should be started at a very young age, repeated intravenous injections can be problematic even in some adults, haemostasis is less effective in inhibitor patients, there is no universal treatment regimen, compliance and adherence to treatment can be a problem especially in adolescents, and prevention of arthropathy in children with inhibitors is more problematic (14).

\section{New treatment approaches}

New products are needed in the field of haemophilia care to decrease costs (more competitors), to facilitate prophylaxis (e.g. by development of long-acting molecules), to improve haemostatic efficacy (modified molecules with enhanced activity) and to reduce inhibitor development (modified epitope sites, non-replacement therapies). Gene therapy is also an important goal. New products with unmodified pharmacokinetic properties are Turoctocog alfa, a B-domain truncated FVIII (Novo Nordisk), BAY 81-8973, a full-length protein-free FVIII (Bayer), Simoctocog alfa, a B-domaindeleted FVIII produced by a human cell line (Octapharma), CSL-627, a single-chain FVIII with higher affinity for VWF (CSL Behring), and Nonacog gamma, a full-length product (Baxter Healthcare Corporation).

Various technologies have been used in an attempt to extend the half-life of factor VIII. These are Fc fusion, albumin fusion, GlycoPEGylation and site-specific PEGylation (15). With these products, it is possible to maintain the same trough level by reducing the frequency of infusions or a higher trough level can be achieved by maintaining the same dosing interval as in standard regimens with unmodified products. The first approach can be advantageous in patients with poor venous access or low compliance to intensive 
regimens, and the latter may confer a better (joint) protection especially in individuals with an active lifestyle and who play sports. In the B-LONG study of rFIXFc in previously treated patients (PTPs), the half-life was $82.1 \mathrm{~h}$, and in the tailored prophylaxis arm, the median dosing interval was 14 d. No inhibitors were detected (16). In the A-LONG phase 3 study of rFVIIIFc in PTPs, the half-life was approximately $19 \mathrm{~h}$ and $30 \%$ of subjects achieved a 5-d dosing interval. No inhibitors were detected (17). Results from studies involving previously untreated patients (PUPs) are awaited: both rFIXFc and rFVIIIFc (Biogen/Sobi) are produced using a human cell line.

Other new treatments are represented by non-replacement therapies that enhance blood coagulation by different mechanisms. All these new molecules are undergoing clinical trials.

One example is ACE910/Emicizumab, a bispecific recombinant humanised antibody able to bind FIXa and FX so mimicking the cofactor function of FVIII. It is effective both in patients with or without inhibitors, lasts for up to a month in plasma and can be given subcutaneously once weekly to maintain a haemostatic effect able to prevent spontaneous bleeding (18).

Another approach is represented by the inhibition of inhibitors of coagulation as the use of anti-TFPI antibodies as Concizumab (Novo Nordisk), which targets tissue factor pathway inhibitor (TFPI). In a phase 1, multicentre, randomised, double-blind placebo-controlled trial of 24 patients without inhibitors, no inhibitors developed during the study and the product showed a favourable safety profile after either subcutaneous or intravenous administration (19).

Another way to enhance coagulation activation is by modulating the activity of natural inhibitors. ALN-AT3/Fitusiran is a synthetic GalNAc-conjugated RNA interference therapeutic molecule that is designed to suppress liver production of antithrombin mRNA. It is given by subcutaneous injection once monthly. It results in a $50 \%$ reduction in circulating antithrombin with a weekly $0.125 \mathrm{mg} / \mathrm{kg}$ dosing (20). A phase 1 trial in patients with haemophilia $\mathrm{A}$ and $\mathrm{B}$ is currently underway $(21,22)$.

Haemophilia is an ideal candidate for gene therapy as it is a monogenic disease. Several clinical trials are underway (five in haemophilia B and one in haemophilia A). In haemophilia B, there are promising results, with sustained FIX expression following AAV-mediated gene transfer. However, there are still some obstacles to be overcome, which include the presence of pre-existing anti-AAV antibodies, and the difficulty in scaling up vector manufacturing.

In summary, all novel investigative therapies are associated with potential risks and benefits that need to be proven. Fewer injections, higher troughs and subcutaneous delivery could represent the future of haemophilia therapy. Gene therapy still needs to be optimised. Safety concerns and difficulties with laboratory monitoring may limit the introduction of new therapies in clinical practice. Pharmacoeconomic profiling of new therapies may become a strong factor influencing their introduction in specific subsets of patients.

\section{Debate 2 - What are the best treatments for the future?}

Maria Elisa Mancuso (Milan, Italy)

As new technologies become reality, the range of treatment options available to haemophilia treatment centre will expand. This means that members of the multidisciplinary team will need to make decisions as to which option they think is the best treatment for their patients. During this session, delegates discussed the benefits and limitations of these treatment options, formulating arguments for why each could be their therapy of choice in the future. The treatment options that were discussed were as follows:

1 Newer factor concentrates (e.g. extended half-life products, increased activity products);

2 Treatments with novel targets (e.g. anti-TFPI antibodies, bispecific antibodies to FIXa and FX, antithrombin knockdown);

3 Gene editing (e.g. wild-type clotting factor gene insertion);

4 Conventional factor concentrates/other.

\section{Newer factor concentrates (e.g. extended half-life products, increased activity products)}

These are the best option because they suit young children in that they do not have to be given so often, thus avoiding frequent venous access. They are also very useful for sporty patients, helping to keep trough levels high. However, they are costly and cannot yet be given subcutaneously. Caution needs to be exercised in elderly patients in whom the trough level may reach such a level as to cause a risk of thrombosis, although this can be minimised by frequent laboratory monitoring of FVIII levels. When used to provide cover during surgery, the products do not have to be given so frequently and may be associated with a reduced hospital stay.

\section{Treatments with novel targets (e.g. anti-TFPI antibodies, bispecific antibodies to FIXa and FX, antithrombin knockdown)}

The advantage of treatments with novel targets is that they will lead to individualised treatment for patients, which is good for those with difficult venous access because they are delivered subcutaneously. However, they have been designed as prophylactic agents and not to be used in the case of acute bleeding. They will also help in spending healthcare budgets wisely. They may require specific laboratory assays and safety might be a concern although no side effects have been reported so far. Further information about the products 
is required before they can be recommended for widespread use.

\section{Gene editing (e.g. antithrombin knockdown, clotting factor gene insertion)}

Gene therapy intended as the possibility to produce normal amounts of clotting factors would represent the cure and hence the best option for patients with haemophilia, but there are more questions than answers. For FIX, gene therapy has been going on for decades, and FIX concentrate use has been reduced by $90 \%$ in phase $2 / 3$ trials (23). For FVIII, trials are at a phase 1 stage but it has been reported that two patients were in excess of $50 \%$ for FVIII expression (24). Gene therapy is an interesting area in development; however, once it has been administered to a patient, it cannot be reversed, and the many unknowns around long-term safety and efficacy represent a big concern.

\section{Conventional factor concentrates/other}

The advantages of the conventional factor concentrates are that their efficacy is predictable, and there is a multitude of evidence for their safety and efficacy in different clinical settings including major surgery There is flexibility in how you can administer the product and standard laboratory test to monitor efficacy. Production can be increased if necessary and the products are available globally, although some lowincome countries, such as Bangladesh and some countries in Latin America, have great difficulties in obtaining the products and they still use cryoprecipitate.

\section{Plenary session 3 - Lifetime management of our patients with haemophilia}

\section{Growing up with haemophilia - from care to coaching}

\section{Pia Petrini (Stockholm, Sweden)}

Young patients with haemophilia live in fear of having a bleed, and they do not like injections. But as they grow older, they take on the feeling that having haemophilia is nothing special and they can live a near-normal life if they take their prophylaxis. There are various challenges in haemophilia treatment beside costs. These include difficulties with venous access and the risk of inhibitor development for babies, independence and adherence during the adolescent years, the difficult decision about knowing when to stop targeted prophylaxis in adulthood, and comorbidities and the increased need for prophylaxis in elderly patients.

Early prophylaxis, started after the first joint bleed or before the age of $2 \mathrm{yr}$, leads to prevention/reduction of joint bleeding, prevention of inhibitor development and prevention of life-threatening bleeds (25). Prophylaxis should be optimised by bleeding pattern, guidance by pharmacokinetics and individualised. Unfortunately, in developing countries, it has to be low dose due to funding constraints but it can still be effective.

To assess the progression of clinical symptoms over time, tools include the annual bleeding rate score, Haemophilia Joint Health Score (HJHS) and ultrasound. To assess the outcome in preventive and clinical trials, MRI, quality of life tools and number of days lost from school/work are used.

With regard to prophylaxis regimen, from the PedNet Registry study reported by Nijdam et al., complete prevention of joint bleeds was found to be most effective on the full regimen (32\% full prophylaxis from start vs. $27 \%$ when the patient was stepped up to full prophylaxis and $8 \%$ phenotype) although at the cost of using most central venous access devices (88\% full vs. $34 \%$ stepped up and $22 \%$ phenotype) (26).

To reduce the risk of inhibitor development when initiating prophylaxis, treatment should be initiated at a low dose, that is usually $25 \mathrm{IU} / \mathrm{kg}$, peak treatment moments should be avoided, no concomitant immunological danger signals and treatment should be given as prophylaxis and not on-demand to avoid low-titre antibodies (27).

The dosage regimen of long-acting FVIII and FIX products can be altered according to how many peaks and troughs are required (28). The effect of half-life on trough levels questions the logic of Monday, Wednesday and Friday dosing and suggests a role for innovative regimens including low-dose daily treatment, which leads to either higher trough levels or decreased FVIII requirement.

Adolescents (aged 10-19 yr) are a special group in terms of haemophilia management. This is a time of rapid physical and psychological (cognitive and emotional) growth and development during which new capacities are developed. It is also a time of changing social relationships, expectations, roles and responsibilities. During this time, there is a transition in activities for self-management (Fig. 1; 29).

Challenges that the healthcare team need to manage during this time in a patient's life are as follows: risk-taking, novelty seeking, sensation seeking and emotionally influenced behaviour without consideration of future outcomes or consequences.

\section{Adulthood and cardiovascular issues in haemophilia}

\section{Pål André Holme (Oslo, Norway)}

The life expectancy of PWH has increased dramatically over the years to that approaching the normal population. In a study by Manco-Johnson et al. (30), it was found that routine prophylaxis with recombinant FVIII led to a significant reduction in bleeding compared to on-demand treatment. However, with the increased life expectancy has come an increase in comorbidities in older $\mathrm{PWH}$, which 


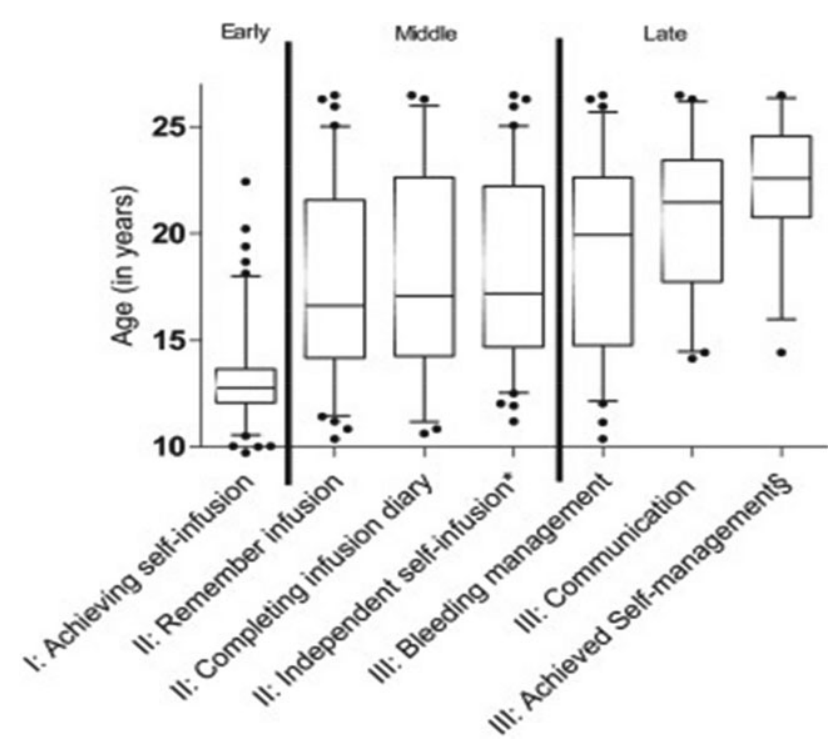

Figure 1 Activities for the self-management process according to age (29).

have not been seen before. The prevalence of hypertension found in older PWH was $45 \%$ according to one study, and haematuria and poor renal function were also problems (31). Acute coronary syndrome (ACS) and myocardial revascularisation should be managed promptly by a multidisciplinary team that includes a haemophilia expert. Each CCC for adult PWH should have a formal clinical referral pathway with a cardiology centre with an emergency unit and 24-h availability of percutaneous coronary intervention (PCI). PCI should be performed as soon as possible under adequate clotting factor protection. Bare metal stents are preferred to drug-eluting stents. Anticoagulants should only be used in PWH after replacement therapy (32). With regard to antiplatelet therapy, the use of GP IIb/IIIa antagonists may be considered in PWH on replacement therapy when clinically indicated. The use of dual antiplatelet therapy including loading doses of clopidogrel prior to PCI may be considered in PWH substituted to high factor levels. Depending on the severity of the bleeding disorder, extended dual antiplatelet therapy after ACS can at least be considered. Minimum trough levels should not fall below $5-15 \%$ in PWH on dual antiplatelet therapy. Trough levels should be $\sim 50 \%$ within $24 \mathrm{~h}$ of an invasive procedure. The duration of dual antiplatelet therapy after ACS and PCI should be reduced to a minimum and should follow the recommendations for patients on vitamin $\mathrm{K}$ antagonists. $\mathrm{PWH}$ receiving antiplatelet therapy should be offered gastric protection.

Minimum trough levels of FVIII/FIX have been suggested depending on the particular situation, for example cardioversion or during transoesophageal echocardiography (33). To calculate the risk of stroke in atrial fibrillation, the CDA2DS2-VASc score [congestive heart failure/left ventricular dysfunction, hypertension, age $\geq 75 \mathrm{yr}$, diabetes mellitus, stroke, TIA/thromboembolism, vascular disease (prior myocardial infarction, peripheral artery disease or aortic plaque), age 65-74 years and sex category (female)] Score can be used (34). The results of the score dictate the treatment that should be given, for example a score $\geq 2$ = oral anticoagulation.

Another score that is used is the HAS-BLED (hypertension, abnormal renal and liver function, stroke, bleeding, labile international normalised ratio, elderly, drugs or alcohol) score to calculate the risk of bleeding for patients on anticoagulation for atrial fibrillation (35).

To summarise, PWH diagnosed with atrial fibrillation should be treated by a multidisciplinary team. The use of vitamin $\mathrm{K}$ antagonists may be considered for $\mathrm{PWH}$ at high risk of stroke with a high enough trough clotting factor level. Cardioversion in a patient with severe haemophilia requires a therapeutic dose of heparin, but should only be carried out after administration of clotting factor.

\section{Haemophilia - one disease, a lifetime of symptoms}

\section{Debra Pollard (London, UK)}

Haemophilia is one disease, but is associated with different challenges and symptoms depending on their 'haemophilia generation'. This includes when they were born and what access they have had to effective treatment throughout their lifespan, how severe their bleeding phenotype and what comorbidities exist, as well as socio-economic factors and their family culture and beliefs. In $30 \%$ of boys, there is no family history of the disease, and they may have had a difficult path to diagnosis including, for some, investigations by social services for non-accidental injury. For mothers of boys with haemophilia, it may be difficult for them to return to full-time work as it may be difficult to find others willing to take on the responsibility of caring for a child with haemophilia; this can have a financial impact on the whole family. As the child grows, there are other issues to be overcome, such as the impact of bleeds on education and social life, and the influence of haemophilia on relationships within the family. During adolescence, the young person needs to establish their own identity and to balance pressure from family/peers/media. It is often a time of challenge, rebellion and confusion as the young person balances the emotional, social and physical changes. For the adolescent, it is particularly hard if their peers are engaging in sports or activities that they are not able to participate in. Planning for school trips, parties and sleepovers presents additional challenges that may mean they have to disclose their haemophilia to others at a time in their life when they do not wish to be seen as 'different'. Most adolescents just want to be 'normal', whatever that may mean to them. For older teenagers, drinking alcohol or other risk-taking behaviour 
when out with friends can lead to accidents that may result in bleeding. Such patients may feel pressured to join in to avoid bullying by their peers, and they may be prone to anxiety and depression.

In adulthood, the key issues for PWH are further education and training, travel difficulties, employment concerns, maintaining health and treatment and finance.

In addition to these expected challenges, the healthcare team must always expect the unexpected, in terms of surgical and dental interventions, and the need to develop pathways of care with partners in other areas, such as orthopaedics, cardiology and oncology.

For ageing PWH, the healthcare team faces challenges associated with a lifetime of musculoskeletal bleeds, plus multiple comorbidities alongside the inevitable consequences encountered in the older population, which can lead to a reduced quality of life, social isolation, depression and anxiety.

When reviewing the needs of $\mathrm{PWH}$, it can be seen that they require complex assessment and intervention throughout life from all parts of an adaptable multidisciplinary team working in partnership with patients and families.

\section{Plenary Session 4 - Applying genetics in a clinical setting: issues for carriers of haemophilia}

\section{Roseline d'Oiron (Paris, France)}

For women in families with haemophilia patients, there are two important questions. The first is whether they are carrying the affected gene, which can only be answered by carrying out an appropriate genetic test. The second is whether as carriers they, too, are at risk of bleeding. Indeed, in our practice, we do find carriers of haemophilia A or B with low levels of FVIII or FIX $(<40 \mathrm{IU} / \mathrm{dL})$, respectively, which is equivalent to levels seen in patients with mild haemophilia and which must be treated accordingly (36). However, ambiguous names are used frequently, mostly using a genetic designation at the expense of a clinical bleeding disorder.

Non-recognition of female haemophilia can lead to several problems such as:

1 Delay and loss of opportunity for diagnosis and treatment of bleeding episodes;

2 Delayed diagnosis of infectious complications (37);

3 Negative emotional and behavioural responses to medical experiences (38);

4 Haemophilia Treatment Centre not recognised as a suitable place of care;

5 Denial of insurance coverage.

Currently, women with haemophilia are still infrequently included in national databases although some improvement in this has been observed. In the FranceCoag National Registry, the number of haemophilia carriers included has increased by 2.35 -fold over 5 yr.
Increased bleeding tendency seems 1.5-fourfold higher with factor levels $<40 \mathrm{IU} / \mathrm{dL}$ compared to women with factor levels $>60 \mathrm{IU} / \mathrm{dL}$ (39), although other groups have not found such a correlation (40).

Approximately one-third of haemophilia carriers have bleeding episodes, but some have an increased bleeding tendency despite normal factor levels, while others have a normal bleeding tendency with lowered factor levels. One measurement of factor level may not always reflect the lowest level, so assays may need to be repeated. Thrombin generation assay results, although related to FVIII:C levels, did not differ significantly between the carriers with elevated and normal bleeding scores (41).

There has been a progressive improvement of methods of genetic diagnosis. A mutation has been identified in $95 \%$ of patients with severe haemophilia A, in $85-90 \%$ with mild/moderate haemophilia A, and in $99 \%$ with haemophilia B $(42,43)$. Next-generation sequencing will contribute to progressively close at least some of the remaining gaps in our knowledge. So the genetic diagnosis for the majority of families with haemophilia should not be problematic except for some rare specific concerns, such as somatic mosaicism found in $13 \%$ of families with isolated cases of severe haemophilia A, mainly with point mutations (42). However, between $30 \%$ and $90 \%$ of mothers are unaware of their genetic status at their first pregnancy (44). About $50 \%$ of carriers undergo genetic testing only after $25 \mathrm{yr}$ of age. On the other hand, there are concerns about testing children because of fears over the psychosocial effects, threat of stigmatisation or lack of proper informed consent by the child. Therefore, the overall recommended agreement is to wait until the child can give informed consent for a genetic test to be performed, which usually happens at the end of adolescence. Reasons for the delayed genetic diagnosis of carriers include denial and lack of information on the potential benefits of the genetic diagnosis. Awareness of carrier status before pregnancy will ease complex decisions regarding reproductive choices and allow prevention of bleeding complications of the mother and neonate at birth.

Carrier testing can be improved by better liaison between the multidisciplinary care team, the family and patient groups, improved information and development of research.

In conclusion, both men and women may have haemophilia, even if they have different illness experiences, and either can pass on the affected gene.

\section{Plenary session 5 - big data, little data, shared data, private data - how do we make the most of real-life data?}

Jan Blatný (Brno, Czech Republic), Marijke van den Berg (Utrecht, The Netherlands), Hervé Chambost (Marseille, France), Radoslaw Kaczmarek (Wroclaw, Poland)

Real-life data in haemophilia care can come from various sources such as clinical registries and case reports. The 
reason why real-life data need to be considered is that they serve as important feedback that can improve further treatment. Real-life data enable the 'full story' to be told, if properly and responsibly obtained and handled. The data also have the potential to offer additional safety information. The EU Haemophilia Treatment Strategy advocates for the availability of real-life data. The principles of care were outlined and reported by Colvin et al. (4) stating that national registries of patients are recommended within each country, along with collection of treatment statistics.

The two major methods of data collection are (randomised) clinical trials and real-life data. The advantage of collecting data through clinical trials is that there is a precise testing of specific hypotheses/questions, patients are often randomised, there is logistic/technical/statistical support, the protocol is standardised, and safety is monitored. Disadvantages are that patients recruited to the trial are an elected/ limited 'unreal' population. Those patients considered 'too difficult' are often excluded from the trial. Not all possible confounders are necessarily taken into account, and there is a limited time frame for the respective studies. To summarise, we can say that the 'danger of evidence-based medicine' is that it may not reflect the 'real-world' situation/ practice and poorly designed randomised controlled trials could even be harmful. Clinical trials are often also demanding for staff and subjects/families; for example, many blood samples may need to be taken.

In contrast, the benefits of real-life data are that the data are instantly available, there are no exclusion criteria for patients, there is no time limitation, and there is no selection bias (if performed correctly). Data are often 'universal', not product and/or disease specific and can be pooled together. They can be also used for long-term instant 'safety monitoring'. On the other hand, disadvantages of real-life data are less standardisation and completeness in often different databases/structures. Administration and monitoring might be a problem, and lack of resources might be a hindrance. In other words, randomised controlled trials focus on efficacy, whereas real-life data reflect effectiveness.

As mentioned above, real-life data have the potential to overcome certain limitations of phase III trials, which are as follows: the study period may be too short to capture all relevant effects; too specific to cover all possible related issues/parameters; poor fit for 'real' local population/care; low event rate of studied event; not capture other factors influencing real practice (adherence, etc). To take advantage of real-life data, however, they have to be: meaningful (to answer the questions), standardised (to enable cooperation and processing), valid, safe (to comply with legal and ethical settings) and robust (to minimise the potential biases and to identify potential confounders).

Challenges to be overcome regarding real-life data are the need for 'goodwill', general agreement and the need to share the data with others on a national/international level. Optimal and perhaps standardised parameters will enable their comparison and allow them to be pooled together. Professionalism, data administration and validation, as well as safety of data and the role that patients play, are important factors to take into consideration when dealing with real-life data.

\section{Debate 3 - how do we make the most of real- life data?}

Jan Blatný (Brno, Czech Republic), Marijke van den Berg (Utrecht, The Netherlands), Hervé Chambost (Marseille, France), Radoslaw Kaczmarek (Wroclaw, Poland)

The debate focussed on several key issues related to real-life data. The main reasons for collecting real-life data were considered to be the need for feedback for medical professionals, with such data being required for negotiation with authorities, for scientific reasons and assessment of safety.

With regard to who should collect and process the real-life data, this should be carried out ideally by professional data managers, in either the haemophilia treatment centre, or outsourced. Funding of data collection should be the responsibility of the Government or other official and independent national/international authority. Agreement on standard and standardised parameters set for real-life data reporting on an international level is an important challenge. Existing international databases/registries should be used and adapted to new needs (e.g. EUHASS Registry).

\section{Sharing best practice}

Prior to THE Meeting, treatment centres were invited to submit an abstract, summarising a particular aspect of their experience of haemophilia care. Three of the submitted abstracts were selected for presentation by the Steering Committee, and one treatment centre was awarded THE Meeting Best Practice Award. The three presented abstracts are summarised below.

\section{Practical treatment based on an integrated therapeutic concept}

\section{Susan Halimeh (Duisburg, Germany)}

Haemophilia treatment is very expensive, and there is no remuneration for medical services or optimal medical care for PWH. Over recent years, different models to increase the achievement of a suitable standard of care for patients with hard to treat diseases such as haemophilia have been discussed in Germany. The health-political targets have been expressed in the changes of the Code of Social Law number $\mathrm{V}$ (SGB V) and in innovations in statutory health insurance. This new legal base provides opportunities to implement innovative treatment concepts outside university hospitals 
and paves the way for ambulant haemophilia centres to offer an integral care package, all encompassed by a legal contract.

The haemophilia CCC has a multidisciplinary team encompassing laboratory staff, motion analysis, nutrition education, etc. Our contract with insurance company BARMER GEK took $2 \mathrm{yr}$ of negotiation until it was signed. During this time, we needed to monitor time management and cost management for the patient's treatment. A financial economist carried out the necessary calculations. The contract was designed to improve medical services, especially for patients in the peripheral regions, and the remuneration was independent of the quantity of factor concentrate. The contract could be terminated from both sides at any time, and also by the insured person. All patients with factor deficiency could participate.

The advantages for the patient are that they get a dental cleaning appointment three times a year, transportation costs to the centre are paid by the insurance company, and physiotherapy is also paid for. In addition, they get a social worker and psychological care, as well as orthopaedic/haematological consultations every week.

The advantages for the insurance company are that there is maximal transparency for prescribing factor concentrate, and price negotiations are in the hand of the insurance company (no influence on kind of factor or quantity of factor). In addition, through intensive care, every bleed will be detected and treated and will be well documented.

Since 1 January 2009, statutory health insurance has been funded via the Health Fund, which is operated at the Federal Insurance Office as a special fund of the Federation. The health fund is financed by premium income that comes from the health insurance funds.

The morbidity-orientated RSE (Morbi RSE) examines morbidity risks directly, using 80 selected disease groups via pseudonymised inpatient and outpatient diagnoses. Data on prescribed pharmaceuticals are used to secure outpatient diagnosis.

From 2012 to 2013, we investigated whether there was an additional benefit from our healthcare model. We found that physiotherapy prevented bleeds and increased physical function, as a result of which the amount of factor concentrate required decreased. However, there is a lack of well-trained physiotherapists specialised in caring for patients with haemophilia and, as a result of which, the HaemAcademy was initiated to train motivated physiotherapists in the special care of patients with haemophilia in Germany (Fig. 2).

The Coagulation Centre Rhine-Ruhr is an example of how haemophilia treatment in accordance with guidelines and with the latest results of international research can be realised in an ambulatory network. Top quality, effective treatment of haemophilia requires an integrated therapeutic concept and the excellent cooperation of an interdisciplinary team.
The HaemAcademy differentiates treatment strategies according to age group (toddlers, children, teenager, adults and older patients) with practical examples. Disease-related background information is provided.

The HaemAcademy can be organised in almost every haemophilia centre in the world, like we did in Cairo.

For the future, we would like to optimise joint function by trying to reduce bleeds, using tailored physiotherapy and tailored FVIII/FIX therapy. The tailored physiotherapy comprises manual therapy (improving range of motion), whereas gait training comprises improving motor deficits in different parts of the gait cycle as measured by motion analysis.

\section{Joint disease surveillance programme in patients with haemophilia: Experience of a Portuguese paediatric tertiary care centre}

\section{Raquel Maia (Lisbon, Portugal)}

Joint bleeds are a major cause of morbidity in patients with haemophilia and may result in severe and chronic arthropathy.

Although primary prophylaxis drastically reduced the number of joint bleeds in children with severe haemophilia (from 20 to $30 / y r$ to $0-1.1 / y r$ ) (45), a few still occur, as well as subclinical events that may result in articular damage.

The use of validated tools is essential to detect minor joint disease (early) and standardise evaluations.

The HJHS, created in 2003, is a physical examination assessment tool that can detect subtle early signs of joint damage. It can be used to monitor joint change over time and to assess the efficacy of treatment regimens in children aged 4-18 yr with haemophilia and mild joint impairment (e.g. as seen in those treated with prophylaxis).

At our paediatric tertiary care centre (Lisbon), a Multidisciplinary Joint Disease Surveillance Program involving a paediatric haematology specialist, a physical medicine and rehabilitation specialist and a dedicated nurse, was

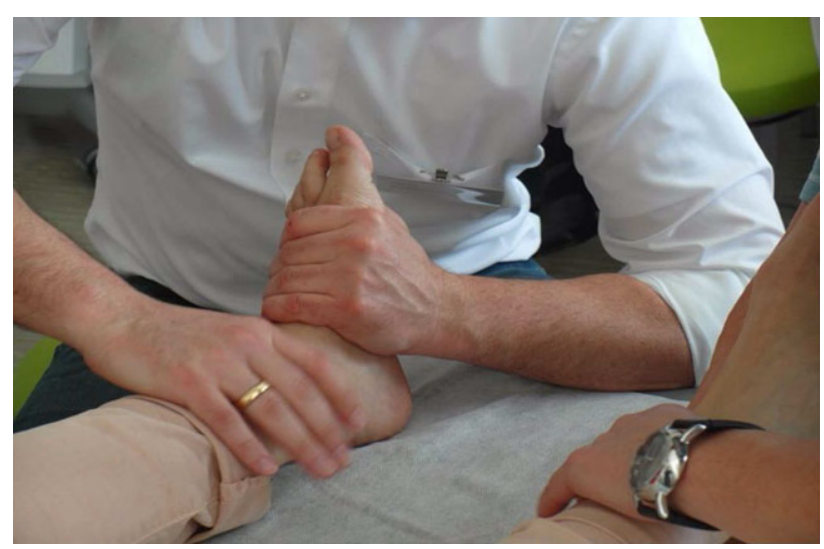

Figure 2 Specialist haemophilia physiotherapist administering treatment to a patient under the HaemAcademy scheme. 
implemented in September 2014 to systematically evaluate and optimise follow-up.

The goals of this programme included the following: (i) to ensure at least one complete joint status evaluation annually or every 6 months of all patients with haemophilia, using the HJHS; (ii) to improve communication between paediatric haematologists and physiatrists/physiotherapists; (iii) to identify at an early stage minor joint abnormalities and adjust the treatment regimen/rehabilitation programme accordingly.

From 23 patients with severe haemophilia, 19 have already attended the multidisciplinary consultation (all over 5 yr old); median age 12 yr (5-19 yr old).

All are on prophylaxis (median age of initiation: 21 months), except for one who is undergoing immune tolerance induction; average HJHS score was $3(37 \%$ have a HJHS of 0); $53 \%$ have joint impairment; mean joint score was 2 (the ankle being the most commonly affected joint).

Although the global score was low, similarly to recent studies, most children revealed some degree of joint impairment (with the ankle being most frequently affected).

The detection of minor joint impairment led to treatment regimen adjustments and personalised training programmes (physical therapy/home exercise).

Additionally, this multidisciplinary approach optimised existing resources creating a stronger and more prepared multidisciplinary team; promoted communication between healthcare professionals and allowed for a reduction in the number of medical appointments and work/school absenteeism; improved the awareness of physiatrists/physiotherapists to the disease and the commitment to the patients leading to cooperation beyond the consultation period (e.g. management of bleedings during the acute/subacute period).

The addition of systematic ultrasound assessment to the surveillance is in progress - a dedicated radiologist will collaborate.

\section{Changes in practice to promote bone health in patients with haemophilia (winning abstract)}

\author{
Sayma Raza (Oxford, UK) and (Stephanie Taylor, Oxford, \\ $U K)$
}

As the haemophilia population ages, there is an increase in comorbidities such as osteoporosis. Potential risk factors include vitamin D deficiency, HIV and hepatitis C infection, low body mass index and low activity levels. For men, secondary risk factors for osteoporosis are smoking, excess alcohol consumption, low body mass index, hyperthyroidism and hypogonadism. Following research, recommendations are to test blood $25(\mathrm{OH}) \mathrm{D}$ levels in all patients with haemophilia, assess calcium intake, FRAX (fracture risk assessment tool) score (46) if the patient is over $40 \mathrm{yr}$, and all patients who have a fragility facture to discuss with the osteoporosis service and commence treatment.
Lifestyle and dietary advice includes encouraging the patient to increase regular exercise, reduce high alcohol intake, take vitamin D supplements, optimise calcium intake, maximise sun exposure and adopt fall prevention strategies to reduce fracture risk.

In summary, to reduce the risk of developing osteoporosis and osteoporotic fractures, healthcare staff should encourage patients to take steps to promote bone health, should identify and screen those patients with risk factors associated with low bone mineral density, and if they are confirmed by DEXA to have low bone mineral density and diagnosed with osteoporosis, they should commence treatment with bisphosphonates.

\section{Concluding remarks}

\section{Erik Berntorp (Malmö, Sweden)}

The aim of this second THE Meeting was to promote an integrated approach to delivering the best care in the lifetime of PWH through the education of all members of the multidisciplinary team. The participants came from 20 different countries and included all members of the multidisciplinary team - doctors, nurses and data management personnel - as well as representatives from patient organisations. A range of topics was covered including lifetime patient management, strategies and cost of care, the use of real-life data and the management of haemophilia across Europe. Interesting discussions and exchange of ideas took place at the four interactive workshops, and throughout the meeting, some unmet clinical needs were identified.

\section{Acknowledgements}

Publication of this supplement was supported by an unrestricted educational grant from Sobi. Writing support was provided by Ros Kenn, Freelance Medical Editor/Writer and funded by Sobi.

\section{Disclosures}

Erik Berntorp has provided consultancy/advisory boards/ lecturing/research support to Bayer, Baxalta, Biogen, CSL/ Behring, Grifols, Novo Nordisk, Pfizer, Sobi, Octapharma.

Yesim Dargaud has received grants/research support from Bayer, Baxter, Novo Nordisk, CSL Behring, LFB, Pfizer, LeoPharma, Octapharma; and honoraria from Bayer, Baxter, Novo Nordisk, CSL Behring, Sobi and Octapharma.

Daniel Hart has received grants/research support from Octapharma and Bayer, and consultation fees from Octapharma, Pfizer, Baxter, Novo Nordisk, Uniqure, Roche and Bayer.

Sébastien Lobet has received grants/research support from Pfizer, WFH and Bayer, and consultation fees from Pfizer, Baxalta, Novo Nordisk, Sobi and Bayer.

Maria Elisa Mancuso has acted as a consultant for Bayer Healthcare, Baxalta, CSL Behring, Sobi/Biogen and Pfizer 
and as a speaker for Bayer Healthcare, Sobi/Biogen, Novo Nordisk, CSL Behring and Pfizer.

Roseline d'Oiron has received consulting fees from Baxter, Novo Nordisk, Bayer, Pfizer, Sobi, LFB and CSL Behring.

David Perry has received grants/research support from Baxter, consultation fees from Biogen and has taken part in a speaker's bureau for Novo Nordisk.

Debra Pollard has acted as a consultant for Sobi/Biogen, Bayer, Novo Nordisk and CSL Behring and as a speaker for Sobi/Biogen, Bayer, Novo Nordisk, CSL Behring and Baxalta/ Shire.

Marijke van den Berg has received unrestricted research support from CSL Behring, Novo Nordisk, Wyeth, Baxter and Bayer.

Jan Blatný has received consultation fees from Baxalta/ Shire and Bayer and has taken part in a speaker's bureau for Novo Nordisk, Baxalta/Shire, Pfizer, Sobi and LFB.

Hervé Chambost has no disclosures to declare.

Andrea Doria has no disclosures to declare.

Pål André Holme has received grants/research supports from Bayer, Baxalta, Pfizer and Octapharma; and honoraria or consultation fees from Bayer, Baxalta, Pfizer, Sobi, Grifols and Octapharma.

Radoslaw Kaczmarek has no relevant disclosures to declare.

Lorenzo Mantovani has no disclosures to declare.

Paul McLaughlin has received honoraria from Bayer, Novo Nordisk, Sobi and Baxter.

Lochana Nanayakkara has received honoraria from Pfizer.

Pia Petrini has received honoraria from Novo Nordisk,

Baxalta, Bayer, Octapharma and Pfizer.

Thomas Sannié has no disclosures to declare.

Edward Laane has no disclosures to declare.

Raquel Maia has no relevant disclosures to declare.

Athina Dettoraki has no relevant disclosures to declare.

Anna Farrell has no disclosures to declare.

Sébastien Lobet has received honoraria from Bayer,

Baxalta, Novo Nordisk, Pfizer, Sobi and CSL; and has participated in a company-sponsored speaker's bureau for Novo Nordisk and Pfizer.

Susan Halimeh has received speaker honorarium from Bayer Healthcare $\mathrm{GmbH}$, Baxalta Innovations $\mathrm{GmbH}$, Biotest AG, CSL Behring GmbH, Novartis Pharma GmbH, Novo Nordisk Pharma GmbH, Octapharma GmbH, LFB GmbH and Pfizer Pharma Gmbh.

Sayma Raza has no disclosures to declare.

Stephanie Taylor has no disclosures to declare.

\section{References}

1. France WG, Wolf P. Treatment and prevention of chronic haemorrhagic arthropathy and contractures in haemophilia. J Bone Joint Surg Br 1965;47:247-55.
2. O'Mahony B, Noone D, Giangrande PL, et al. Haemophilia care in Europe - a survey of 35 countries. Haemophilia 2013;19:e239-47.

3. Windyga J, Grabarczyk P, Stefańska E, Buczma A, Szczepanik AB, Klukowska A, Mikulska M, Medyńska J, Brojer E. [Prevalence of HCV, HBV and HIV infections among severe Polish haemophiliacs]. Przegl Epidemiol 2008;62:415-23. [Article in Polish].

4. Colvin BT, Astermark J, Fischer K, Gringeri A, Lassila R, Schramm W, Thomas A, Ingerslev J; Inter Disciplinary Working Group. European principles of haemophilia care. Haemophilia 2008;14:361-74.

5. Gringeri A, Mantovani LG, Scalone L, Mannucci PM, COCIS Study Group. Cost of care and quality of life for patients with hemophilia complicated by inhibitors: the COCIS Study Group. Blood 2003;102:2358-63.

6. Scalone L, Mantovani LG, Mannucci PM, Gringeri A; COCIS Study Investigators. Quality of life is associated to the orthopaedic status in haemophilic patients with inhibitors. Haemophilia 2006;12:154-62.

7. Schramm W, Gringeri A, Ljung R, et al. Haemophilia care in Europe: the ESCHQoL study. Haemophilia 2012;18: 729-37.

8. Keshava SN, Gibikote S, Doria AS. Imaging evaluation of hemophilia: musculoskeletal approach. Semin Thromb Hemost 2015;41:880-93.

9. Doria AS, Keshava SN, Mohanta A, et al. Diagnostic accuracy of ultrasound for assessment of hemophilic arthropathy: MRI correlation. AJR Am J Roentgenol 2015;204:W336-47.

10. Lauersen JB, Bertelsen DM, Andersen LB. The effectiveness of exercise interventions to prevent sports injuries: a systematic review and meta-analysis of randomised controlled trials. Br J Sports Med 2014;48:871-7.

11. Negrier C, Seuser A, Forsyth A, Lobet S, Llinas A, Rosas M, Heijnen L. The benefits of exercise for patients with haemophilia and recommendations for safe and effective physical activity. Haemophilia 2013;19:487-98.

12. Kalsi H, Nanayakkara L, Pasi KJ, Bowles L, Hart DP. Access to primary dental care for patients with inherited bleeding disorders. Haemophilia 2012;18:510-5.

13. Srivastava A, Brewer AK, Mauser-Bunschoten EP, et al. Guidelines for the management of hemophilia. Haemophilia 2013;19:e1-47.

14. Carcao M. Changing paradigm of prophylaxis with longer acting factor concentrates. Haemophilia 2014;20(Suppl. 4):99-105.

15. Mahdi AJ, Obaji SG, Collins PW. Role of enhanced half-life factor VIII and IX in the treatment of haemophilia. $\mathrm{Br} \mathrm{J}$ Haematol 2015;169:768-76.

16. Powell JS, Pasi KJ, Ragni MV, et al. Phase 3 study of recombinant factor IX Fc fusion protein in hemophilia B. $N$ Engl J Med 2013;369:2313-23.

17. Mahlangu J, Powell JS, Ragni MV, et al and the A-LONG Investigators. Phase 3 study of recombinant factor VIII Fc fusion protein in severe hemophilia A. Blood 2014;123:31725 . 
18. Muto A, Yoshihashi K, Takeda M, et al. Anti-factor IXa/X bispecific antibody (ACE910): hemostatic potency against ongoing bleeds in a hemophilia A model and the possibility of routine supplementation. J Thromb Haemost 2014;12:206-13.

19. Chowdary P, Lethagen S, Friedrich U, et al. Safety and pharmacokinetics of anti-TFPI antibody (concizumab) in healthy volunteers and patients with hemophilia: a randomized first human dose trial. J Thromb Haemost 2015;13:743-54.

20. Sehgal A, Barros S, Ivanciu L, et al. An RNAi therapeutic targeting antithrombin to rebalance the coagulation system and promote hemostasis in hemophilia. Nat Med 2015;21:492-7.

21. ClinicalTrials.gov. A Phase 1 Study of an Investigational Drug, ALN-AT3SC, in healthy volunteers and hemophilia A or B patients. NCT02035605. Available at: https://clinicaltria ls.gov/ct2/show/NCT02035605 (accessed 1 August 2016).

22. ClinicalTrials.gov. An Open-label Extension Study of an Investigational Drug, ALN-AT3SC, in patients with moderate or severe hemophilia A or B. NCT02554773. Available at: https://clinical trials.gov/ct2/show/NCT02554773 (accessed 1 August 2016).

23. Nienhuis AW, Nathwani AC, Davidoff AM. Gene therapy for hemophilia. Hum Gene Ther 2016;27:305-8.

24. BioMarin. BioMarin provides encouraging preliminary data on first 8 patients in hemophilia A gene therapy program. Available at: http://investors.bmrn.com/releasedetail.cfm? releaseid=965945 (accessed 30 September 2016).

25. Petrini P, Valentino LA, Gringeri A, Re WM, Ewenstein B. Individualizing prophylaxis in hemophilia: a review. Expert Rev Hematol 2015;8:237-46.

26. Nijdam A, Kurnik K, Liesner R, Ljung R, Nolan B, Petrini P, Fischer K; PedNet study group. How to achieve full prophylaxis in young boys with severe haemophilia A: different regimens and their effect on early bleeding and venous access. Haemophilia 2015;21:444-50.

27. Gouw SC, van den Berg HM, Fischer K, et al. Intensity of factor VIII treatment and inhibitor development in children with severe hemophilia A: the RODIN study. Blood 2013;121:4046-55.

28. Collins PW, Fischer K, Morfini M, Blanchette VS, Björkman $\mathrm{S}$; International Prophylaxis Study Group Pharmacokinetics Expert Working Group. Implications of coagulation factor VIII and IX pharmacokinetics in the prophylactic treatment of haemophilia. Haemophilia 2011;17:2-10.

29. Schrijvers L, derBeijlevelt-Van Zande M, Peters M, Lock J, Cnossen M, Schuurmans M, Fischer K. Achieving self-management of prophylactic treatment in adolescents: the case of haemophilia. Patient Educ Couns 2016;99:1179-83.

30. Manco-Johnson MJ, Kempton CL, Reding MT, et al. Randomized, controlled, parallel-group trial of routine prophylaxis vs. on-demand treatment with sucrose-formulated recombinant factor VIII in adults with severe hemophilia A (SPINART). J Thromb Haemost 2013;11:1119-27.

31. Holme PA, Combescure C, Tait RC, Berntorp E, Rauchensteiner $\mathrm{S}$, De Moerloose P, on behalf of the Advance Working Group. Hypertension, haematuria and renal functioning in haemophilia a cross-sectional study in Europe. Haemophilia 2016;22:248-55.
32. Staritz P, deMoerloose P, Schutgens R, Dolan G; ADVANCE Working Group. Applicability of the European Society of Cardiology guidelines on management of acute coronary syndromes to people with haemophilia - an assessment by the ADVANCE Working Group. Haemophilia 2013; 19:833-40.

33. Schutgens RE, Klamroth R, Pabinger I, Dolan G; ADVANCE working group. Management of atrial fibrillation in people with haemophilia-a consensus view by the ADVANCE Working Group. Haemophilia 2014;20:e417-20.

34. Lip GY, Nieuwlaat R, Pisters R, Lane DA, Crijns HJ. Refining clinical risk stratification for predicting stroke and thromboembolism in atrial fibrillation using a novel risk factorbased approach: the Euro heart survey on atrial fibrillation. Chest 2010;137:263-72.

35. Pisters R, Lane DA, Nieuwlaat R, de Vos CB, Crijns HJ, Lip GY. A novel user-friendly score (HAS-BLED) to assess 1year risk of major bleeding in patients with atrial fibrillation: the Euro Heart Survey. Chest 2010;138:1093-100.

36. Mauser-Bunschoten EP. Symptomatic carriers of haemophilia. WFH monograph December 2008, No.46.

37. Peynet J, Doncarli A, Rothschild C, et al. Viral infection status of 1036 women with inherited coagulation factor deficiencies within the Francecoag network. Haemophilia 2012;18 (Suppl. 3):1-208, abstract PO-MO-267.

38. Renault NK, Howell RE, Robinson KS, Greer WL. Qualitative assessment of the emotional and behavioural responses of haemophilia A carriers to negative experiences in their medical care. Haemophilia 2011;17:237-45.

39. Plug I, Mauser-Bunschoten EP, Bröcker-Vriends AH, van Amstel HK, van der Bom JG, van Diemen-Homan JE, Willemse J, Rosendaal FR. Bleeding in carriers of hemophilia. Blood 2006;108:52-6.

40. Miesbach W, Alesci S, Geisen C, Oldenburg J. Association between phenotype and genotype in carriers of haemophilia A. Haemophilia 2011;17:246-51.

41. Olsson A, Hellgren M, Berntorp E, Holmström M, Baghaei F. Bleeding phenotype in carriers of haemophilia A does not correlate with thrombin generation. Haemophilia 2015;21:e111-3.

42. Leuer M, Oldenburg J, Lavergne JM, Ludwig M, Fregin A, Eigel A, Ljung R, Goodeve A, Peake I, Olek K. Somatic mosaicism in hemophilia A: a fairly common event. Am J Hum Genet 2001;69:75-87.

43. Kasper CK, Lin JC. Prevalence of sporadic and familial haemophilia. Haemophilia 2007;13:90-2.

44. Kessler L, Adams R, Mighion L, Walther S, Ganguly A. Prenatal diagnosis in haemophilia A: experience of the genetic diagnostic laboratory. Haemophilia 2014;20:e384-91.

45. Fischer K, Collins P, Björkman S, Blanchette V, Oh M, Fritsch S, Schroth P, Spotts G, Ewenstein B. Trends in bleeding patterns during prophylaxis for severe haemophilia: observations from a series of prospective clinical trials. Haemophilia 2001;17:4533-8.

46. FRAX WHO Fracture risk assessment score. Available at: https://www.shef.ac.uk/FRAX/tool.jsp (accessed 5 August 2016). 